\title{
Nonlinear Equations of Infinite Order Defined by an Elliptic Symbol
}

\author{
Mauricio Bravo Vera \\ Departamento de Matemática y Ciencia de la Computación, Universidad de Santiago de Chile, Casilla 307, Correo 2, Santiago, Chile \\ Correspondence should be addressed to Mauricio Bravo Vera; mauricio.bravo.v@usach.cl
}

Received 7 February 2014; Accepted 21 April 2014; Published 8 May 2014

Academic Editor: A. Zayed

Copyright (C) 2014 Mauricio Bravo Vera. This is an open access article distributed under the Creative Commons Attribution License, which permits unrestricted use, distribution, and reproduction in any medium, provided the original work is properly cited.

\begin{abstract}
The aim of this work is to show existence and regularity properties of equations of the form $f(\Delta) u=U(x, u(x))$ on $\mathbb{R}^{n}$, in which $f$ is a measurable function that satisfies some conditions of ellipticity and $\Delta$ stands for the Laplace operator on $\mathbb{R}^{n}$. Here, we define the class of functions to which $f$ belongs and the Hilbert space in which we will find the solution to this equation. We also give the formal definition of $f(\Delta)$ explaining how to understand this operator.
\end{abstract}

\section{Introduction}

This paper is motived by recent researches in string theory and cosmology where the equations appear with infinitely many derivatives [1-13]. For example, we can mention the following equation:

$$
p^{a \partial_{t}^{2}} \phi=\phi^{p}, \quad a>0
$$

where $p$ is a prime number. This equation describes the dynamics of the open $p$-adic string for the scalar tachyon field (see $[4,7,8,10-12]$ and the references therein). To consider this equation as an equation in an infinite number of derivatives, we can formally expand the left-hand side as a power series in $\partial_{t}^{2}$. Let us note that, in the articles [10, 11], (1) has already been studied via integral equation of convolution type and it is worth mentioning that in the limit $p \rightarrow 1$ this equation becomes the local logarithmic Klein-Gordon equation [14-16].

Another common example of an equation with infinitely many derivatives that is worth pointing out corresponds to the dynamical equation of the tachyon field in bosonic open string field theory that can be set as

$$
\left[(1+\square) e^{-c \square}-2\right] \phi=\phi^{2},
$$

where $\square=-\partial_{t}^{2}+\Delta$ is the d'Alembertian operator (see [17]).
In the present paper, our aim is to show existence and regularity of solutions for nonlinear equations of infinite order of type

$$
f(\Delta) u=U(\cdot, u),
$$

where the operator $f(\Delta)$ is defined in terms of Laplacian over $\mathbb{R}^{n}$ and the function $u$ is defined in whole Euclidean space $\mathbb{R}^{n}$. First, we define the class of functions to which the symbol $f$ belongs. This class, as we will see, contains symbols that are from a very general kind and in general do not belong to the classic Hörmander class defined to pseudodifferential operators [18].

It is worth pointing out that this paper is inspired by the articles [19-21], where the authors work out this type of equations. In the article [19], the authors consider the operator $f(\Delta)$ acting on whole Euclidean space $\mathbb{R}^{n}$ or over a compact Riemannian manifold $(M, g)$ and show the existence and regularity of solutions for certain values of a constant $\beta>n / 2$, where $n>1$, which will be defined in detail in Section 2. In the present paper, assuming that the nonlinearity $U$ satisfies a Lipschitz type inequality, we extend these results and show the existence and uniqueness of solutions for (3) for values of $\beta>0$.

This work is organized as follows: in Section 2, definitions and basic properties about the class of functions to which the symbol $f$ belongs are given. Furthermore, we introduce the vector space where we will seek the solution to nonlinear 
equation (3). At the end of this section, the definition of operator $f(\Delta)$ and an embedding lemma are given. In Section 3, we solve the linear equation

$$
f(\Delta) u+u=g
$$

where $g \in L^{2}\left(\mathbb{R}^{n}\right)$, obtaining also some properties that the solutions of this equation have and which will be useful to solve the nonlinear equation in the following section. Finally in Section 4, using Banach's fixed point theorem, we show existence and uniqueness of the solution to the nonlinear problem (3).

\section{Preliminaries}

The aim of this section is to define and develop some basic aspects that will be needed in the study of the nonlinear equation

$$
f(\Delta) u=U(x, u(x))
$$

defined in terms of the "symbol $f$ " First, we give sense to operator $f(\Delta)$. For this purpose, we have to clearly define the conditions that should be satisfied by the symbol $f$. Thus, we give the following definition.

Let us consider measurable functions $f$ such that the following two conditions are verified:

(P) the function $s \mapsto f\left(-s^{2}\right)$ is nonnegative;

$\left(E_{\beta}\right)$ there exist real numbers $\beta, M$, and $R>0$ such that

$$
M\left(1+|\xi|^{2}\right)^{\beta / 2} \leq f\left(-|\xi|^{2}\right) \quad \forall \xi \text { with }|\xi|>R \text {. }
$$

If a measurable function satisfies the above two conditions, we will say that $f$ belongs to the class $\mathscr{G}^{\beta}$ or simply that $f$ is a $\mathscr{G}^{\beta}$-symbol. Although the above condition $\left(E_{\beta}\right)$ coincides with the condition of ellipticity given for pseudodifferential operators (see [22]), we can highlight that these symbols are not defined symbols in the sense of Hörmander [18].

Now, from the definition of class $\mathscr{G}^{\beta}$, we obtain the following propositions.

Proposition 1. Let $\beta, \delta>0$ be fixed. If $f \in \mathscr{G}^{\beta}$ and $g \in \mathscr{G}^{\delta}$, then $f \cdot g \in \mathscr{G}^{\beta+\delta}$.

Proof. It is clear that the function $f \cdot g$ satisfies condition (P). On the other hand, since $f$ and $g$ satisfy condition $\left(E_{\beta}\right)$, we have that there exist positive constants $M_{1}, M_{2}, \beta, \delta, R_{1}$, and $R_{2}$ such that

$$
\begin{array}{ll}
M_{1}\left(1+|\xi|^{2}\right)^{\beta / 2} \leq f\left(-|\xi|^{2}\right) & \forall|\xi|>R_{1} \\
M_{2}\left(1+|\xi|^{2}\right)^{\delta / 2} \leq g\left(-|\xi|^{2}\right) & \forall|\xi|>R_{2} .
\end{array}
$$

Multiplying both inequalities, we obtain that

$$
M\left(1+|\xi|^{2}\right)^{(\beta+\delta) / 2} \leq f \cdot g\left(-|\xi|^{2}\right) \quad \forall|\xi|>R,
$$

where $M=M_{1} M_{2}$ and $R=\max \left\{R_{1}, R_{2}\right\}$. Therefore $f \cdot g \in$ $\mathscr{G}^{\beta+\delta}$.
Proposition 2. If $0<\delta<\beta$, then $\mathscr{G}^{\beta} \subset \mathscr{G}^{\delta}$.

Proof. Since $1<\left(1+|\xi|^{2}\right)$ and $\delta<\beta$, then $\left(1+|\xi|^{2}\right)^{\delta / 2}<$ $\left(1+|\xi|^{2}\right)^{\beta / 2}$. On the other hand, let $f \in \mathscr{G}^{\beta}$; then $f$ satisfies condition $(\mathrm{P})$ and there exist real numbers $M, \beta$, and $R$ such that for all $\xi$ with $|\xi|>R$ we have that

$$
M\left(1+|\xi|^{2}\right)^{\delta / 2}<M\left(1+|\xi|^{2}\right)^{\beta / 2} \leq f\left(-|\xi|^{2}\right)
$$

thus $f \in \mathscr{G}^{\delta}$.

Lemma 3. Let $r>0$ be fixed, and let $f_{r}(s)=(1-s)^{r / 2}-1$. Then $f_{r} \in \mathscr{G}^{\beta}$ for all $\beta \leq r$.

Proof. Clearly the function $s \mapsto f_{r}(-s)=\left(1+s^{2}\right)^{r / 2}-1 \geq 0$.

Now, in order to see that $f_{r}$ satisfies the condition $\left(E_{\beta}\right)$, let us note that, for $\beta<r$, we have

$$
\begin{aligned}
f_{r}\left(-|\xi|^{2}\right) & =\left(1+|\xi|^{2}\right)^{r / 2}-1 \\
& \geq\left(1+|\xi|^{2}\right)^{\beta / 2}-1 \\
& =\left(1+|\xi|^{2}\right)^{\beta / 2}\left[1-\frac{1}{\left(1+|\xi|^{2}\right)^{\beta / 2}}\right]
\end{aligned}
$$

Let us note that, in the right-hand side of the above equality,

$$
\lim _{|\xi| \rightarrow \infty}\left(1-\frac{1}{\left(1+|\xi|^{2}\right)^{\beta / 2}}\right)=1 .
$$

Therefore, for $M_{r}<1$ fixed, there exists $R_{r}>0$ such that, for all $\xi$ with $|\xi|>R_{r}$, we have

$$
M_{r}\left(1+|\xi|^{2}\right)^{\beta / 2} \leq f_{r}\left(-|\xi|^{2}\right)
$$

Finally, we have $f_{r} \in \mathscr{G}^{\beta}$ for $\beta \leq r$.

Next, we introduce the vector space where we will find the solution to our nonlinear equation.

Definition 4. Given $\beta>0$ and the symbol $f$ in the class $\mathscr{G}^{\beta}$ fixed, one defines the space $\mathscr{H}^{\beta}(f)$ as the set of complex valued functions $g$ defined on $\mathbb{R}^{n}$ such that $g$ is measurable, its Fourier transform $\mathscr{F}(g)$ exists, and

$$
\int_{\mathbb{R}^{n}}\left[1+f\left(-|\xi|^{2}\right)\right]^{2}|\mathscr{F}(g)(\xi)|^{2} d \xi<\infty
$$

We can endow $\mathscr{H}^{\beta}(f)$ with the following inner product. Given $g_{1}, g_{2} \in \mathscr{H}^{\beta}(f)$,

$$
\left\langle g_{1}, g_{2}\right\rangle=\int_{\mathbb{R}^{n}}\left[1+f\left(-|\xi|^{2}\right)\right]^{2} \mathscr{F}\left(g_{1}\right)(\xi) \overline{\mathscr{F}\left(g_{2}\right)(\xi)} d \xi,
$$

and with this definition, the vector space $\mathscr{H}^{\beta}(f)$ turns out to be a Hilbert space. Moreover, from the definition of $\mathscr{H}^{\beta}(f)$ 
and Plancherel's theorem, we have that $\mathscr{H}^{\beta}(f) \hookrightarrow L^{2}\left(\mathbb{R}^{n}\right)$ since

$$
\begin{aligned}
\|u\|_{L^{2}\left(\mathbb{R}^{n}\right)} & =\|\mathscr{F}(u)\|_{L^{2}\left(\mathbb{R}^{n}\right)} \\
& =\int_{\mathbb{R}^{n}}|\mathscr{F}(u)(\xi)|^{2} d \xi \\
& <\int_{\mathbb{R}^{n}}\left[1+f\left(-|\xi|^{2}\right)\right]^{2}|\mathscr{F}(u)(\xi)|^{2} d \xi \\
& =\|u\|_{\mathscr{C}^{\beta}(f)} .
\end{aligned}
$$

Proposition 5. Let $f_{r}$ be defined as in Lemma 3. Then a function $u \in \mathscr{H}^{r}\left(f_{r}\right)$ if and only if $u \in H^{r}\left(\mathbb{R}^{n}\right)$.

Proof. First, let us note that

$$
1+\left(f_{r}\left(-|\xi|^{2}\right)\right)=\left(1+|\xi|^{2}\right)^{r / 2}=\langle\xi\rangle^{r}
$$

In the last equality, we have used the notation given by Taylor [23]. Next, by the definition of $\mathscr{H}^{\beta}(f)$, we have that $u \in$ $\mathscr{H}^{r}\left(f_{r}\right)$ if and only if there exist its Fourier transform $\mathscr{F}(u)$ and $\left(1+f_{r}\left(-|\xi|^{2}\right)\right) \mathscr{F}(u) \in L^{2}\left(\mathbb{R}^{n}\right)$.

This is equivalent, as we have seen above, to

$$
\langle\xi\rangle^{r} \mathscr{F}(u) \in L^{2}\left(\mathbb{R}^{n}\right),
$$

and this is equivalent to $u \in H^{r}\left(\mathbb{R}^{n}\right)$.

We now introduce the definition of operator $f(\Delta)$. The reason why we will consider this definition comes from a formal computation; see, for instance, [19].

Definition 6. For a $\mathscr{G}^{\beta}$-symbol $f$, one defines the operator $f(\Delta)$ as follows:

$$
f(\Delta) u=\mathscr{F}^{-1}\left(f\left(-|\xi|^{2}\right) \mathscr{F}(u)(\xi)\right) .
$$

Analogously, we can define the linear operator $L=f(\Delta)+$ Id as

$$
L u=\mathscr{F}^{-1}\left(\left(1+f\left(-|\xi|^{2}\right)\right) \mathscr{F}(u)(\xi)\right), \quad\left(u \in \mathscr{H}^{\beta}(f)\right)
$$

It is easy to see that $L$ acts on $\mathscr{H}^{\beta}(f)$ and for all $u \in \mathscr{H}^{\beta}(f)$ we have that $L u \in L^{2}\left(\mathbb{R}^{n}\right)$.

Lemma 7. Let $f \in \mathscr{G}^{\beta}$; then

(1) for $s \in \mathbb{R}$ and $s \leq \beta$, the embedding $\mathscr{H}^{\beta}(f) \hookrightarrow H^{s}\left(\mathbb{R}^{n}\right)$ holds;

(2) for all $k \geq 1$ such that $n / 2+k<s \leq \beta$, the embedding $\mathscr{H}^{\beta}(f) \hookrightarrow C^{k}\left(\mathbb{R}^{n}\right)$ holds.

\section{The Linear Equation}

In this section, we will consider the linear operator $L$ defined in (19). We solve the linear equation

$$
L u=g,
$$

where $g \in L^{2}\left(\mathbb{R}^{n}\right)$. Furthermore, we establish certain regularity properties that enjoy the solutions of the linear equation (20).

Theorem 8. Let $f$ be in the class $\mathscr{G}^{\beta}$. Then, for each $g \in$ $L^{2}\left(\mathbb{R}^{n}\right)$, there exists a unique solution $u_{g} \in \mathscr{H}^{\beta}(f)$ to linear equation (20). Moreover, the equality

$$
\left\|u_{g}\right\|_{\mathscr{H}^{\beta}(f)}=\|g\|_{L^{2}\left(\mathbb{R}^{n}\right)}
$$

holds.

Proof. By the definition of operator $L$ given in (19), we have that the equation $L u=g$ is equivalent to

$$
\mathscr{F}^{-1}\left(\mathscr{F}(u)(\xi)+f\left(-|\xi|^{2}\right) \mathscr{F}(u)(\xi)\right)=g .
$$

Now, since $g \in L^{2}\left(\mathbb{R}^{n}\right)$, we can apply Fourier transform to both sides of the above identity, obtaining that

$$
\begin{gathered}
\mathscr{F}(u)(\xi)+f\left(-|\xi|^{2}\right) \mathscr{F}(u)(\xi)=\mathscr{F}(g)(\xi) \\
\left(1+f\left(-|\xi|^{2}\right)\right) \mathscr{F}(u)(\xi)=\mathscr{F}(g)(\xi) \\
\mathscr{F}(u)(\xi)=\frac{\mathscr{F}(g)(\xi)}{1+f\left(-|\xi|^{2}\right)} .
\end{gathered}
$$

Applying the inverse Fourier transform to both sides of this equality, we find the explicit form of $u$

$$
u=\mathscr{F}^{-1}\left(\frac{\mathscr{F}(g)(\xi)}{1+f\left(-|\xi|^{2}\right)}\right) .
$$

Hence $u_{g}=\mathscr{F}^{-1}\left(\mathscr{F}(g)(u) /\left(1+f\left(-|\xi|^{2}\right)\right)\right)$ is the unique solution of the linear equation $L u=g$. In addition, we have that

$$
\left(1+f\left(-|\xi|^{2}\right)\right) \mathscr{F}(u)(\xi)=\mathscr{F}(g)(\xi)
$$

then

$$
\int_{\mathbb{R}^{n}}\left[1+f\left(-|\xi|^{2}\right)\right]^{2}|\mathscr{F}(u)(\xi)|^{2} d \xi=\|\mathscr{F}(g)\|_{L^{2}\left(\mathbb{R}^{n}\right)} .
$$

But then, from the definition of $\mathscr{H}^{\beta}(f)$ and Plancherel's theorem, we have that $u_{g} \in \mathscr{H}^{\beta}(f)$ and

$$
\left\|u_{g}\right\|_{\mathscr{H}^{\beta}(f)}=\|g\|_{L^{2}\left(\mathbb{R}^{n}\right)^{*}}
$$


Now, in the following two propositions we will show that some extra properties of $g$ will imply additional regularity of $u$.

Proposition 9. Let $f \in \mathscr{G}^{\beta}$, consider the linear equation $L u=g$, defined in (19), and let $g \in L^{2}\left(\mathbb{R}^{n}\right)$. If, in addition, $g \in \mathscr{H}^{\delta}(h)$ for some $\delta>0$ for some $h \in \mathscr{G}^{\delta}$, then the solution $u \in \mathscr{H}^{\delta}(h) \cap \mathscr{H}^{\beta}(f) \cap \mathscr{H}^{\beta+\delta}(f \cdot h)$.

Proof. As we have seen, if $u$ is the solution to the linear equation (20), then

$$
\left(1+f\left(-|\xi|^{2}\right)\right)^{2}|\mathscr{F}(u)(\xi)|^{2}=|\mathscr{F}(g)(\xi)|^{2}
$$

holds. Multiplying both sides of the above equation by $(1+$ $\left.h\left(-|\xi|^{2}\right)\right)^{2}$ and integrating over $\mathbb{R}^{n}$, we have obtained

$$
\begin{aligned}
& \int_{\mathbb{R}^{n}} {\left[\left(1+h\left(-|\xi|^{2}\right)\right)\left(1+f\left(-|\xi|^{2}\right)\right)\right]^{2}|\mathscr{F}(u)(\xi)|^{2} d \xi } \\
&=\|g\|_{\mathscr{C}^{\delta}(h)}<\infty .
\end{aligned}
$$

Since $1 \leq\left(1+f\left(-|\xi|^{2}\right)\right)^{2}$, then we have that

$$
\begin{aligned}
& \int_{\mathbb{R}^{n}}\left(1+h\left(-|\xi|^{2}\right)\right)^{2}|\mathscr{F}(u)(\xi)|^{2} d \xi \\
& \leq \int_{\mathbb{R}^{n}}\left[\left(1+h\left(-|\xi|^{2}\right)\right)\left(1+f\left(-|\xi|^{2}\right)\right)\right]^{2}|\mathscr{F}(u)(\xi)|^{2} d \xi \leq \infty ;
\end{aligned}
$$

thus, $u \in \mathscr{H}^{\delta}(h)$.

On the other hand, since $\left(1+h \cdot f\left(-|\xi|^{2}\right)\right) \leq(1+$ $\left.h\left(-|\xi|^{2}\right)\right)\left(1+f\left(-|\xi|^{2}\right)\right)$, we have

$$
\begin{aligned}
& \int_{\mathbb{R}^{n}}\left[1+h \cdot f\left(-|\xi|^{2}\right)\right]^{2}|\mathscr{F}(u)(\xi)|^{2} d \xi \\
& \leq \int_{\mathbb{R}^{n}}\left[\left(1+h\left(-|\xi|^{2}\right)\right)\left(1+f\left(-|\xi|^{2}\right)\right)\right]^{2}|\mathscr{F}(u)(\xi)|^{2} d \xi<\infty .
\end{aligned}
$$

Therefore, $u \in \mathscr{H}^{\beta+\delta}(f \cdot h)$.

Now, we will show that if the function $g$ in (20) is invariant under rotations, then the solution $u$ will be invariant under rotations too.

Proposition 10. If $g$ is invariant under rotations, that is, for each rotation, $R \in S O(n)$ and for all $x \in \mathbb{R}^{n}, g(R x)=g(x)$ holds, then the solution $u$ to the linear equation $L u=g$ is invariant under rotation as well.

Proof. Suppose that $g(R x)=g(x)$ where $R \in S O(n)$; then let us note that the Fourier transform of $g$ is invariant under rotation too. Indeed,

$$
\begin{aligned}
\mathscr{F}(g)(R \xi) & =\int_{\mathbb{R}^{n}} e^{-i(R \xi) \cdot y} g(y) d y \\
& =\int_{\mathbb{R}^{n}} e^{-i \xi \cdot\left(R^{-1} y\right)} g(y) d y
\end{aligned}
$$

$$
\begin{aligned}
& =\int_{\mathbb{R}^{n}} e^{-i \xi \cdot y} g(R y) d y \\
& =\int_{\mathbb{R}^{n}} e^{-i \xi \cdot y} g(y) d y=\mathscr{F}(g)(\xi) .
\end{aligned}
$$

Hence,

$$
\begin{aligned}
u(R x) & =\int_{\mathbb{R}^{n}} \frac{e^{i(R x) \cdot \xi} \mathscr{F}(g)(\xi)}{1+f\left(-|\xi|^{2}\right)} d \xi \\
& =\int_{\mathbb{R}^{n}} \frac{e^{i x \cdot\left(R^{-1} \xi\right)} \mathscr{F}(g)(\xi)}{1+f\left(-|\xi|^{2}\right)} d \xi \\
& =\int_{\mathbb{R}^{n}} \frac{e^{i x \cdot \xi} \mathscr{F}(g)(R \xi)}{1+f\left(-|\xi|^{2}\right)} d \xi \\
& =\int_{\mathbb{R}^{n}} \frac{e^{i x \cdot \xi} \mathscr{F}(g)(\xi)}{1+f\left(-|\xi|^{2}\right)} d \xi=u(x) .
\end{aligned}
$$

\section{The Nonlinear Equation}

In this section, our aim will be to study the nonlinear equation

$$
f(\Delta) u=U(\cdot, u),
$$

where the nonlinearity is given by the function

$$
U(x, y)=-y+\delta V(x, y)
$$

where $\delta$ is a nonnegative constant. Assuming certain growth condition for the function $V$, we will prove the existence and uniqueness of solution to this equation. For this purpose, our main tool will be Banach's fixed point theorem and the results developed in Section 3.

Theorem 11. Let $f \in \mathscr{G}^{\beta}$. For $\delta>0$, consider the function $U_{\delta}$ defined by

$$
U_{\delta}(x, y)=-y+\delta V(x, y)
$$

where $V$ is a function such that $V(\cdot, 0) \in L^{2}\left(\mathbb{R}^{n}\right)$. If there exists a function $h \in L^{\infty}\left(\mathbb{R}^{n}\right)$, such that the following inequality holds:

$$
\left|V\left(x, y_{1}\right)-V\left(x, y_{2}\right)\right| \leq|h(x)|\left|y_{1}-y_{2}\right|
$$

then for sufficiently small $\delta$, there exists a unique solution $u \in$ $\mathscr{H}^{\beta}(f)$ to problem (34).

Proof. Note that, from condition (37), we have for the function $V$ the following estimate:

$$
\begin{aligned}
|V(x, y)| & \leq|V(x, y)-V(x, 0)|+|V(x, 0)| \\
& \leq|h(x)||y|+|V(x, 0)| .
\end{aligned}
$$


Now, let us see that if $u \in \mathscr{H}^{\beta}(f)$, then the function $\delta V(\cdot, u)$ defined over $\mathbb{R}^{n}$ is a function belonging to $L^{2}\left(\mathbb{R}^{n}\right)$. Indeed,

$$
\begin{aligned}
\|V(\cdot, u)\|_{L^{2}\left(\mathbb{R}^{n}\right)}^{2} & =\int_{\mathbb{R}^{n}}|V(x, u(x))|^{2} d x \\
& \leq \int_{\mathbb{R}^{n}}(|h(x)||u(x)|+|V(x, 0)|)^{2} d x \\
& \leq 2\left(\|h\|_{L^{\infty}\left(\mathbb{R}^{n}\right)}^{2}\|u\|_{L^{2}\left(\mathbb{R}^{n}\right)}^{2}+\|V(\cdot, 0)\|_{L^{2}\left(\mathbb{R}^{n}\right)}^{2}\right) \\
& <\infty .
\end{aligned}
$$

If we consider the function $U$ given by (35), then the nonlinear equation (34) is equivalent to

$$
L u=\delta V(\cdot, u),
$$

in which $L$ is defined by (19). Now, let us define the operator $\mathscr{R}: \mathscr{H}^{\beta}(f) \mapsto \mathscr{H}^{\beta}(f)$ by

$$
\mathscr{R}(u)=\tilde{u},
$$

where $\tilde{u}$ is the unique solution to the linear equation $L \tilde{u}=$ $\delta V(\cdot, u)$

From this, we see that

$$
\begin{aligned}
& L\left(\widetilde{u_{1}}\right)=\delta V\left(\cdot, u_{1}\right), \\
& L\left(\widetilde{u_{2}}\right)=\delta V\left(\cdot, u_{2}\right) .
\end{aligned}
$$

Now, from the linearity of $L$,

$$
L\left(\widetilde{u_{1}}-\widetilde{u_{2}}\right)=\delta\left(V\left(\cdot, u_{1}\right)-V\left(\cdot, u_{2}\right)\right),
$$

and due to Theorem 8 , we have that

$$
\left\|\widetilde{u_{1}}-\widetilde{u_{2}}\right\|_{\mathscr{H}^{\beta}(f)}=\delta\left\|V\left(\cdot, u_{1}\right)-V\left(\cdot, u_{2}\right)\right\|_{L^{2}\left(\mathbb{R}^{n}\right)^{\prime}} .
$$

Then,

$$
\begin{aligned}
\left\|\mathscr{R}\left(u_{1}\right)-\mathscr{R}\left(u_{2}\right)\right\|_{\mathscr{H}^{\beta}(f)} & =\left\|\widetilde{u_{1}}-\widetilde{u_{2}}\right\|_{\mathscr{H}^{\beta}(f)} \\
& =\delta\left\|V\left(\cdot, u_{1}\right)-V\left(\cdot, u_{2}\right)\right\|_{L^{2}\left(\mathbb{R}^{n}\right)},
\end{aligned}
$$

and since

$$
\begin{aligned}
& \left\|V\left(\cdot, u_{1}\right)-V\left(\cdot, u_{2}\right)\right\|_{L^{2}\left(\mathbb{R}^{n}\right)}^{2} \\
& \quad \leq \int_{\mathbb{R}^{n}}|h(x)|^{2}\left|u_{1}(x)-u_{2}(x)\right|^{2} d x \\
& \quad \leq\|h\|_{L^{\infty}\left(\mathbb{R}^{n}\right)}^{2}\left\|u_{1}-u_{2}\right\|_{\mathscr{H}^{\beta}(f)}^{2}
\end{aligned}
$$

we get

$$
\left\|\mathscr{R}\left(u_{1}\right)-\mathscr{R}\left(u_{2}\right)\right\|_{\mathscr{H}^{\beta}(f)} \leq \delta\|h\|_{L^{\infty}\left(\mathbb{R}^{n}\right)}\left\|u_{1}-u_{2}\right\|_{\mathscr{H}^{\beta}(f)} .
$$

Now, if we choose sufficiently small $\delta$, such that $\delta\|h\|_{L^{\infty}\left(\mathbb{R}^{n}\right)}<$ 1 , then we have that $\mathscr{R}$ is a contraction, and by Banach's fixed point theorem, there exists a unique $u_{0} \in \mathscr{H}^{\beta}(f)$ such that

$$
\mathscr{R}\left(u_{0}\right)=u_{0} \text {. }
$$

That is,

$$
L\left(u_{0}\right)=\delta V\left(\cdot, u_{0}\right)
$$

Corollary 12. Let $\beta, f, V$, and $h$ be as in Theorem 11. In addition, suppose that the function $V$ is such that there exist real constants $r, k$ with $r+\beta>n / 2+k$, so that for all $u \in \mathscr{H}^{\beta}(f)$ one has $V(\cdot, u) \in H^{r}\left(\mathbb{R}^{n}\right)$. Then, the solution to nonlinear equation (34) with the nonlinearity (35) belongs to the class $C^{k}\left(\mathbb{R}^{n}\right)$

Proof. Let $\mathscr{R}: \mathscr{H}^{\beta}(f) \rightarrow \mathscr{H}^{\beta}(f)$ be the operator defined in (41). Since $\mathscr{R}$ is acting over $\mathscr{H}^{\beta}(f)$ and for all $u \in \mathscr{H}^{\beta}(f)$ we have $\delta V(\cdot, u) \in H^{r}\left(\mathbb{R}^{n}\right)$, then, by Proposition $5, \delta V(\cdot, u) \in$ $\mathscr{H}^{r}\left(f_{r}\right)$. Subsequently by Proposition 9 , we have that, for all $u \in \mathscr{H}^{\beta}(f), \mathscr{R}(u)=\widetilde{u} \in \mathscr{H}^{\beta+r}\left(f \cdot f_{r}\right)$, as $\beta+r>n / 2+k$, and by Lemma 7, we get $\tilde{u} \in C^{k}\left(\mathbb{R}^{n}\right)$. Therefore, the fixed point of $\mathscr{R}$, that is, the solution to nonlinear equation (34), belongs to the class $C^{k}\left(\mathbb{R}^{n}\right)$.

Let us see the following example.

Example 13. Let $\beta=3$ and $V: \mathbb{R}^{3} \rightarrow \mathbb{R}$ defined by

$$
V\left(x_{1}, x_{2}, x_{3}\right)=\frac{\log \left(1+x_{3}^{2}\right)}{1+\left(x_{1}^{2}+x_{2}^{2}\right)} \text {. }
$$

It is easily seen that this function satisfies the conditions of Theorem 11 and for all $u \in \mathscr{H}^{\beta}(f)$, we have $V(\cdot, u) \in H^{1}\left(\mathbb{R}^{2}\right)$, and then applying Corollary 12 with $\beta=3, r=1$, and $n=$ 2 , we have that $k=2$; therefore, the solution to nonlinear equation (34) with the nonlinearity (50) belongs to the class $C^{2}\left(\mathbb{R}^{2}\right)$

In Theorem 11, if the function $V(x, y)$ satisfies a Lipschitztype condition with respect to the variable $y$, then there exists a unique solution to the nonlinear equation (34). Now, we will see that if we change the global Lipschitz condition to a local Lipschitz condition only, for certain $\delta$, the nonlinear equation has a solution, but we cannot ensure uniqueness.

Theorem 14. Let $f \in \mathscr{G}^{\beta}$ and $\delta>0$. Consider the function $U_{\delta}$ defined by

$$
U_{\delta}(x, y)=-y+\delta V(x, y)
$$

where $V(\cdot, 0) \in L^{2}\left(\mathbb{R}^{n}\right)$. Suppose that, for all $R>0$, there exists $h_{R} \in L^{\infty}\left(\mathbb{R}^{n}\right)$ such that, for all $u_{1}, u_{2} \in B_{L_{2}}(0, R):=\{u \in$ $\left.L^{2}\left(\mathbb{R}^{n}\right):\|u\|_{L^{2}\left(\mathbb{R}^{n}\right)}<R\right\}$, the inequality

$$
\left|V\left(x, u_{1}(x)\right)-V\left(x, u_{2}(x)\right)\right| \leq\left|h_{R}(x)\right|\left|u_{1}(x)-u_{2}(x)\right|
$$

holds. Then, for sufficiently small $\delta$, there exists solution $u \in$ $\mathscr{H}^{\beta}(f)$ to problem (34). 
Proof. Let $u \in B_{L_{2}}(0, R)$; then

$$
|V(x, y)| \leq\left|h_{R}(x)\right||u(x)|+|V(x, 0)|,
$$

and if $u \in \mathscr{H}^{\beta}(f)$, then we have

$$
\begin{aligned}
& \|V(\cdot, u)\|_{L^{2}\left(\mathbb{R}^{n}\right)}^{2} \\
& \quad \leq 2\left\|h_{R}\right\|_{L^{\infty}\left(\mathbb{R}^{n}\right)}^{2}\|u\|_{L^{2}\left(\mathbb{R}^{n}\right)}^{2}+\|V(\cdot, 0)\|_{L^{2}\left(\mathbb{R}^{n}\right)}^{2}<\infty .
\end{aligned}
$$

Now, let us define the following set:

$$
\mathscr{Y}_{\rho}=\left\{u \in \mathscr{H}^{\beta}(f):\|u\|_{\mathscr{C}^{\beta}(f)} \leq \rho\right\}
$$

and the operator $\mathscr{R}_{\rho}: \mathscr{Y}_{\rho} \mapsto \mathscr{Y}_{\rho}$ by $\mathscr{R}_{\rho}(u)=\widetilde{u}$, where $\widetilde{u}$ is the unique solution to the linear equation $L(\widetilde{u})=\delta V(\cdot, u)$.

Let us see that, for all $u \in \mathscr{Y}_{\rho}$, if we consider

$$
\delta<M_{1}=\frac{\rho}{\left(2 \rho\left\|h_{R}\right\|_{L^{\infty}\left(\mathbb{R}^{n}\right)}^{2}+\|V(\cdot, 0)\|_{L^{2}\left(\mathbb{R}^{n}\right)}^{2}\right)^{1 / 2}},
$$

we have $\mathscr{R}_{\rho}(u) \in \mathscr{Y}_{\rho}$, because $\mathscr{R}_{\rho}(u)=\tilde{u}$ is the solution to the linear equation $L(\widetilde{u})=\delta V(\cdot, u)$; then we have

$$
\begin{aligned}
\|\widetilde{u}\|_{\mathscr{H}^{\beta}(f)} & =\delta\|V(\cdot, u)\|_{L^{2}\left(\mathbb{R}^{n}\right)} \\
& \leq \delta\left(2\left\|h_{R}\right\|_{L^{\infty}\left(\mathbb{R}^{n}\right)}^{2}\|u\|_{\mathscr{C}^{\beta}(f)}^{2}+\|V(\cdot, 0)\|_{L^{2}\left(\mathbb{R}^{n}\right)}^{2}\right)^{1 / 2} \\
& \leq \delta\left(2\left\|h_{R}\right\|_{L^{\infty}\left(\mathbb{R}^{n}\right)}^{2} \rho+\|V(\cdot, 0)\|_{L^{2}\left(\mathbb{R}^{n}\right)}^{2}\right)^{1 / 2} \leq \rho .
\end{aligned}
$$

Now, let us note that

$$
\begin{aligned}
\left\|\mathscr{R}_{\rho}\left(u_{1}\right)-\mathscr{R}_{\rho}\left(u_{2}\right)\right\|_{\mathscr{H}^{\beta}(f)} & =\left\|\widetilde{u_{1}}-\widetilde{u_{2}}\right\|_{\mathscr{H}^{\beta}(f)} \\
& \leq \delta\left\|h_{R}\right\|_{L^{\infty}\left(\mathbb{R}^{n}\right)}\left\|u_{1}-u_{2}\right\|_{\mathscr{\ell}^{\beta}(f)} .
\end{aligned}
$$

Next, if we choose $\delta<M_{2}=1 /\left\|h_{R}\right\|_{L^{\infty}\left(\mathbb{R}^{n}\right)}$, we have that $\mathscr{R}_{\rho}$ is a contraction; therefore, choosing $\delta<\min \left\{M_{1}, M_{2}\right\}$ by Banach's fixed point theorem, we have that there exists a unique solution $u \in \mathcal{Y}_{\rho}$ which is a solution to the nonlinear equation (34).

Now, we will see that if the nonlinearity $U$ is radial, that is, invariant under rotations with respect to $x$, then the unique solution to the equation

$$
f(\Delta) u=U(\cdot, u)
$$

is radial as well. To show this, we define the set

$$
\begin{aligned}
& \mathscr{H}^{\beta}(f)_{\text {rad }} \\
& \quad=\left\{u \in \mathscr{H}^{\beta}(f): \forall \text { rotation } R \in \operatorname{SO}(n, \mathbb{R})\right.
\end{aligned}
$$

$$
\text { we have } \left.u(R x)=u(x) \text {, for a.e. in } \mathbb{R}^{n}\right\} \text {. }
$$

Note that $\mathscr{H}^{\beta}(f)_{\text {rad }}$ is a closed set in $\mathscr{H}^{\beta}(f)$. Thus $\mathscr{H}^{\beta}(f)_{\text {rad }}$ is a Hilbert space.

If we consider in $\mathscr{H}^{\beta}(f)_{\mathrm{rad}}$ the metric $d\left(u_{1}, u_{2}\right)=$ $\left\|u_{1}-u_{2}\right\|_{\mathscr{H}^{\beta}(f)}$, we can clearly see that $\left(\mathscr{H}^{\beta}(f)_{\text {rad }}, d\right)$ is a complete metric space.
Theorem 15. Suppose that $f \in \mathscr{G}^{\beta}, \delta>0$ and that the nonlinearity $U$ is invariant under rotations with respect to $x$. Assume also that $V(\cdot, y) \in L^{2}\left(\mathbb{R}^{n}\right)$ and there exists $h \in L^{\infty}\left(\mathbb{R}^{n}\right)$ such that

$$
\left|V\left(x, y_{1}\right)-V\left(x, y_{2}\right)\right|<|h(x)|\left|y_{1}-y_{2}\right| .
$$

Then, for sufficiently small $\delta$, there exists a unique solution $u \in$ $\mathscr{H}^{\beta}(f)_{\text {rad }}$ to the nonlinear equation (34).

Proof. Let $\mathscr{R}_{\mathrm{rad}}: \mathscr{H}^{\beta}(f)_{\mathrm{rad}} \mapsto \mathscr{H}^{\beta}(f)_{\mathrm{rad}}$ be the operator defined by $\mathscr{R}_{\text {rad }}(u)=\widetilde{u}$, where $\tilde{u}$ is the unique solution to the linear equation $L \tilde{u}=\delta V(\cdot, u)$. Since the nonlinearity $U$ is invariant under rotations with respect to $x$, it follows that the function $\delta V(\cdot, u)$ is invariant under rotations, due to Proposition 10 , we have that $\tilde{u}$ is radial, and therefore $\mathscr{R}_{\text {rad }}$ is well defined.

As we have seen,

$$
\begin{gathered}
\left\|\mathscr{R}_{\mathrm{rad}}\left(u_{1}\right)-\mathscr{R}_{\mathrm{rad}}\left(u_{2}\right)\right\|_{\mathscr{H}^{\beta}(f)_{\mathrm{rad}}} \\
\quad=\left\|\mathscr{R}\left(u_{1}\right)-\mathscr{R}\left(u_{2}\right)\right\|_{\mathscr{H}^{\beta}(f)} \\
\leq \delta\|h\|_{L^{\infty}\left(\mathbb{R}^{n}\right)}\left\|u_{1}-u_{2}\right\|_{\mathscr{H}^{\beta}(f)} .
\end{gathered}
$$

Again, choosing $\delta<1 /\|h\|_{L^{\infty}\left(\mathbb{R}^{n}\right)}$, we have that $\mathscr{R}_{\text {rad }}$ is a contraction, and by Banach's fixed point theorem, we have a unique $u \in \mathscr{H}^{\beta}(f)_{\text {rad }}$ that is solution to the nonlinear equation $f(\Delta) u=U(\cdot, u)$.

\section{Conflict of Interests}

The authors declare that there is no conflict of interests regarding the publication of this paper.

\section{Acknowledgments}

This work has been partially supported by Project MECESUP2 PUC0711 and FONDECYT Grant no. 1130554.

\section{References}

[1] N. Barnaby, "A new formulation of the initial value problem for nonlocal theories," Nuclear Physics B, vol. 845, no. 1, pp. 1-29, 2011.

[2] N. Barnaby, T. Biswas, and J. M. Cline, " $p$-adic inflation," Journal of High Energy Physics, vol. 2007, no. 4, article 056, 35 pages, 2007.

[3] N. Barnaby and N. Kamran, "Dynamics with infinitely many derivatives: the initial value problem," Journal of High Energy Physics, vol. 2008, no. 2, article 008, 40 pages, 2008.

[4] N. Barnaby and N. Kamram, "Dynamics with infinitely many derivatives: variable coeficient equations," Journal of High Energy Physics, no. 12, article 022, 27 pages, 2008.

[5] G. Calcagni, M. Montobbio, and G. Nardelli, "Route to nonlocal cosmology," Physical Review D-Particles, Fields, Gravitation and Cosmology, vol. 76, no. 12, Article ID 126001, 20 pages, 2007.

[6] G. Calcagni, M. Montobbio, and G. Nardelli, "Localization of nonlocal theories," Physics Letters, B: Nuclear, Elementary Particle and High-Energy Physics, vol. 662, no. 3, pp. 285-289, 2008. 
[7] B. Dragovich, "Zeta-nonlocal scalar fields," Theoretical and Mathematical Physics, vol. 157, no. 3, pp. 1671-1677, 2008.

[8] N. Moeller and B. Zwiebach, "Dynamics with infinitely many time derivatives and rolling tachyons," Journal of High Energy Physics, vol. 6, no. 10, p. 34, 2002.

[9] W. Taylor, "String field theory", in Approaches to Quantum Gravity, D. Oriti, Ed., pp. 210-228, Cambridge University Press, 2009.

[10] V. S. Vladimirov, "The equation of the p-adic open string for the scalar tachyon field," Izvestiya Mathematics, vol. 69, no. 3, pp. 487-512, 2005.

[11] V. S. Vladimirov and Y. I. Volovich, "Nonlinear dynamics equation in $p$-adic string theory," Teoreticheskaya i Matematicheskaya Fizika, vol. 138, pp. 355-368, 2004.

[12] V. S. Vladimirov, I. V. Volovich, and E. I. Zelenov, P-adic Analysis and Mathematical Physics, World Scientific, Singapore, 1994.

[13] E. Witten, "Non-commutative geometry and string field theory," Nuclear Physics B, vol. 268, no. 2, pp. 253-294, 1986.

[14] K. Bartkowski and P. Górka, "One-dimensional Klein-Gordon equation with logarithmic nonlinearities," Journal of Physics A: Mathematical and Theoretical, vol. 41, Article ID 355201, 11 pages, 2008.

[15] A. A. Gerasimov and S. L. Shatashvili, "On exact tachyon potential in open string field theory," Journal of High Energy Physics, vol. 4, no. 10, p. 34, 2000.

[16] P. Górka, "Logarithmic Klein-Gordon equation," Acta Physica Polonica B, vol. 40, pp. 59-66, 2009.

[17] D. Ghoshal and A. Sen, "Tachyon condensation and brane descent relations in p-adic string theory," Nuclear Physics B, vol. 584, no. 1-2, pp. 300-312, 2000.

[18] L. Hormander, The Analysis of Linear Partial Differential Operators III, Springer, Berlin, Germany, 1985.

[19] P. Górka, H. Prado, and E. G. Reyes, "On a general class of nonlocal equations," Annales Henri Poincaré, vol. 14, pp. 947966, 2013.

[20] P. Górka, H. Prado, and E. G. Reyes, "Functional calculus via Laplace transform and equations with infinitely many derivatives," Journal of Mathematical Physics, vol. 51, no. 10, Article ID 103512, 2010.

[21] P. Górka, H. Prado, and E. G. Reyes, "Nonlinear equations with infinitely many derivatives," Complex Analysis and Operator Theory, vol. 5, no. 1, pp. 313-323, 2011.

[22] M. W. Wong, "On some spectral properties of elliptic pseudodifferential operators," Proceedings of the American Mathematical Society, vol. 99, pp. 683-689, 1987.

[23] M. E. Taylor, Partial Differential Equations I. Basic Theory, Springer, New York, NY, USA, 2010. 


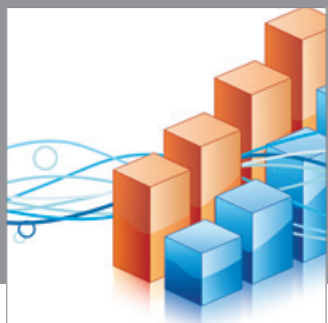

Advances in

Operations Research

mansans

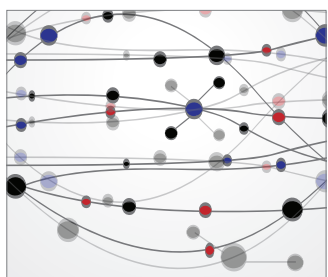

The Scientific World Journal
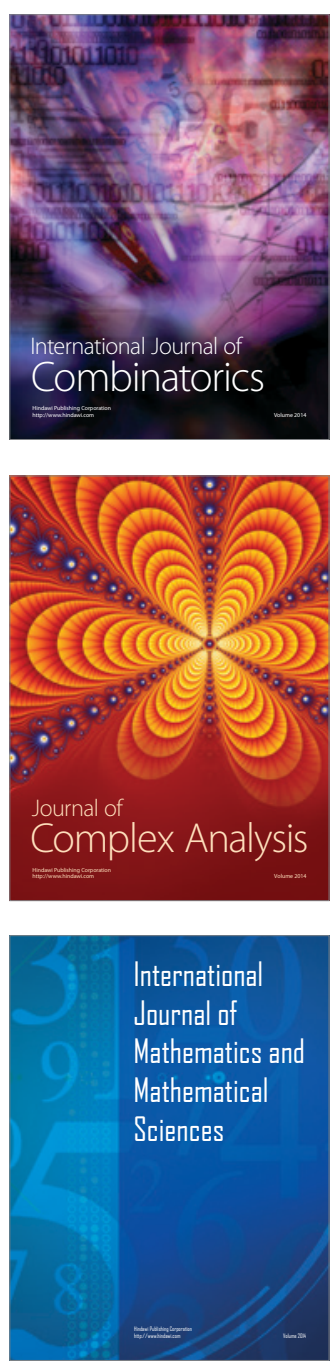
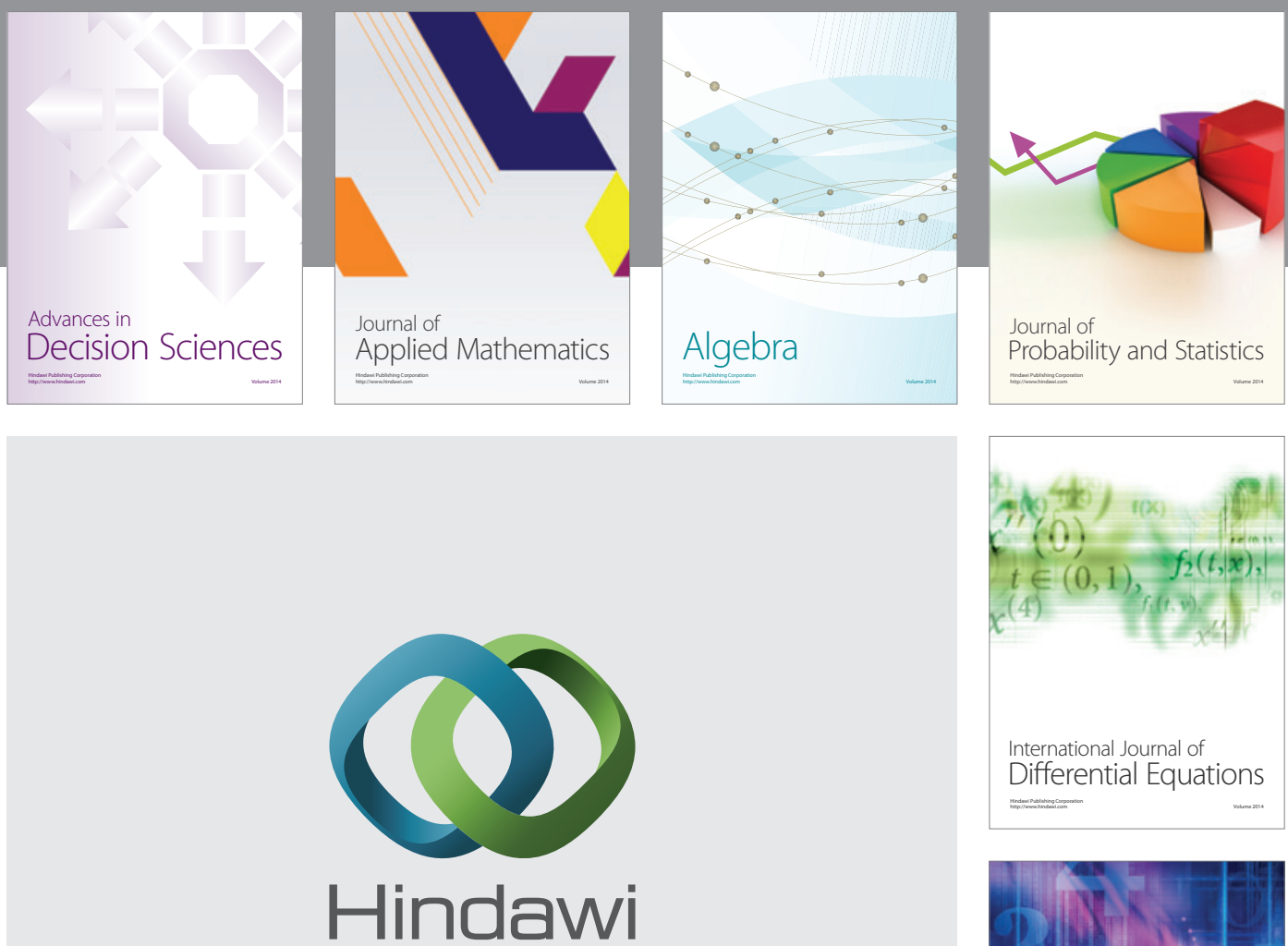

Submit your manuscripts at http://www.hindawi.com
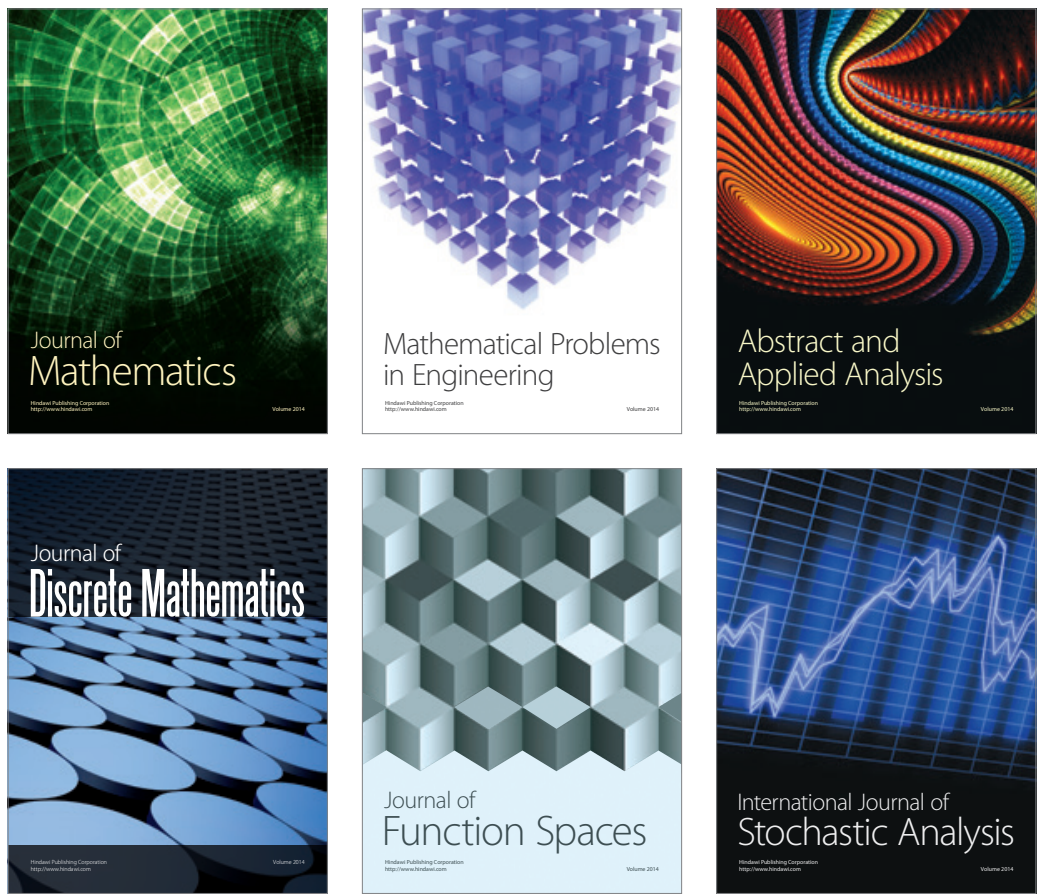

Journal of

Function Spaces

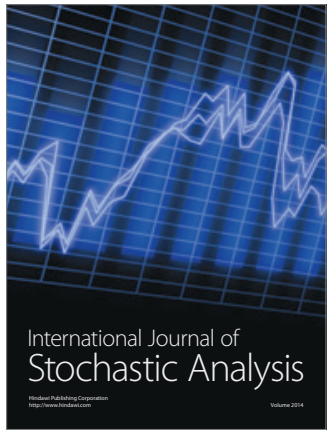

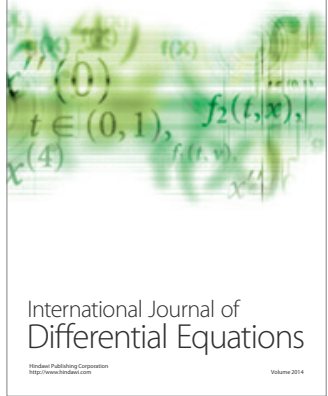
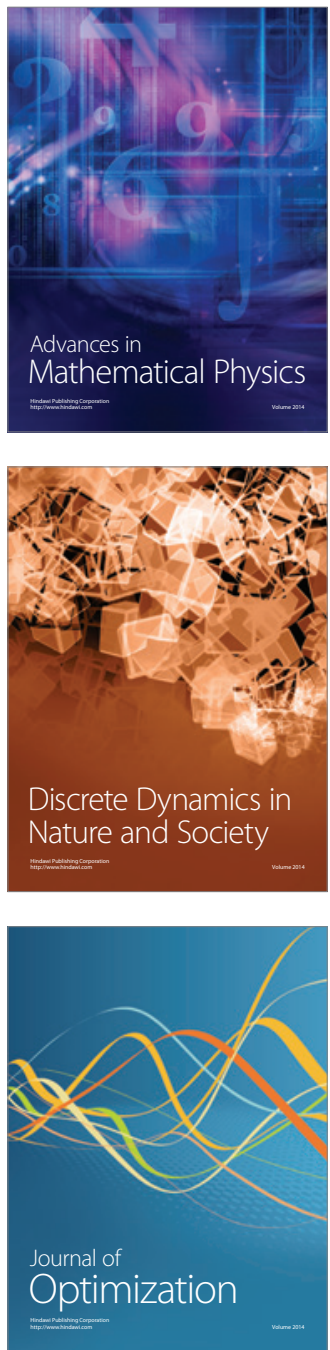